\title{
Remarques sur les fonctions érudites et méditatives de l'eschatologie dans le haut Moyen Âge ibérique
}

\section{Gaelle Bosseman}

\author{
(2) OpenEdition \\ Journals \\ Édition électronique \\ URL : https://journals.openedition.org/cem/17501 \\ DOI : 10.4000/cem. 17501 \\ ISSN : 1954-3093 \\ Éditeur \\ Centre d'études médiévales Saint-Germain d'Auxerre
}

Référence électronique

Gaelle Bosseman, « Remarques sur les fonctions érudites et méditatives de l'eschatologie dans le haut Moyen Âge ibérique », Bulletin du centre d'études médiévales d'Auxerre / BUCEMA [En ligne], 24.2 2020, mis en ligne le 19 décembre 2020, consulté le 22 septembre 2022. URL : http:// journals.openedition.org/cem/17501; DOI : https://doi.org/10.4000/cem.17501

Ce document a été généré automatiquement le 22 septembre 2022.

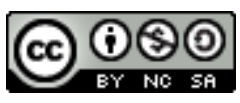

Creative Commons - Attribution - Pas d'Utilisation Commerciale - Partage dans les Mêmes Conditions 4.0 International - CC BY-NC-SA 4.0

https://creativecommons.org/licenses/by-nc-sa/4.0/ 


\title{
Remarques sur les fonctions érudites et méditatives de l'eschatologie dans le haut Moyen Âge ibérique
}

\author{
Gaelle Bosseman
}

1 Le thème des fins dernières occupe une place relativement importante dans les textes latins produits après la conquête islamique de la péninsule Ibérique ${ }^{1}$; pris dans leur ensemble, ceux-ci ont pu paraître significatifs d'un climat d'anxiété apocalyptique. Le succès $\mathrm{du}$ seul commentaire exégétique linéaire composé dans la période, le Commentaire sur l'Apocalypse attribué au moine Beatus de Liébana (v. 776) ${ }^{2}$, semble confirmer cette

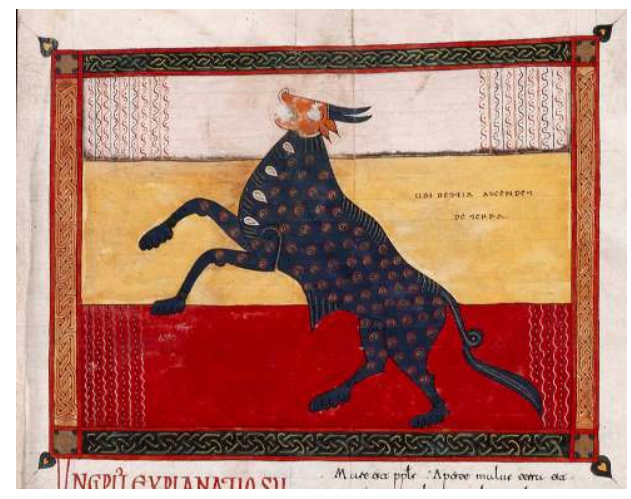
lecture: il serait révélateur selon le philologue Juan Gil d'une «Espagne tourmentée et suspendue à ses espérances eschatologiques $^{3}$ ».

2 Dans le débat sur la réalité des peurs millénaristes à l'approche de l'an 800 et de l'an $1000^{4}$, l'hypothèse d'une croyance adventiste généralisée ne rend toutefois pas compte de la diversité du contenu des sources : de nombreux textes abordent la fin des temps dans une optique sinon érudite ou contemplative, du moins dénuée de toute urgence apocalyptique. Le premier exemple d'une telle relecture concerne le Commentaire de Beatus de Liébana lui-même: il est aujourd'hui généralement admis que le moine s'inscrit dans une compréhension spiritualisée et allégorique de l'Apocalypse héritée de sa source principale, le commentateur africain Tyconius ( $\left(v^{\mathrm{e}}\right.$ siècle) ${ }^{5}$. 
De fait, les textes sur la fin des temps sont des productions savantes. Plutôt que le reflet de peurs ou d'espérances apocalyptiques qui auraient animé les chrétiens après la conquête islamique de 711 , l'eschatologie s'intègre dans des discours aux fonctions multiples, très généralement érudites - compilation de savoirs, discours sur le temps, sur l'histoire humaine, usages polémiques ou encore politiques. Elle y apparaît comme un objet tant de croyance que de savoir. Ce sont ces fonctions érudites et contemplatives - centrales dans la compréhension médiévale de l'eschatologie - que cet article entend explorer.

\section{La constitution d'un savoir sur la fin des temps et ses usages non-apocalyptiques}

4 Connaître ce qui se produira à la fin des temps, la date de celle-ci et ce qu'il adviendra ensuite est un objet de connaissance et de recherche intellectuelle pour les lettrés. Dans ce contexte, et contrairement à l'hypothèse développée par les tenants de l'école des terreurs ${ }^{6}$, les calculs de la date de la fin des temps ne semblent pas révélateurs d'une attente imminente, mais relèvent de fonctions érudites diverses.

5 La fin des temps est l'aboutissement et la borne ultime de l'histoire humaine. Sur le modèle des six jours de la création, celle-ci est conçue depuis Augustin comme une succession de six âges ; l'humanité évolue depuis l'Incarnation du Christ dans le sixième et dernier âge avant la fin du monde ${ }^{7}$. La date de la fin des temps constitue un horizon d'attente sur lequel se sont développées différentes hypothèses; en Occident, depuis Irénée de Lyon, les exégètes ont associé une durée de mille ans à chacun des six âges compris comme un jour pour Dieu dans une interprétation typologique du Psaume $89,4^{8}$. Selon cette lecture largement reprise au haut Moyen Âge, le monde durerait ainsi 6000 ans.

6 Dans un passage maintes fois commenté de son Commentaire sur l'Apocalypse, Beatus de Liébana s'est livré au calcul : en suivant la datation de l'Incarnation fixée en l'an 5200 dans la chronique universelle d'Eusèbe-Jérôme, il conclut que la fin du sixième âge aura lieu en l'an 800. Dans la première édition du texte, achevée en 776, l'âge du monde est ainsi fixé à 5976 ans, il reste vingt-cinq années avant l'échéance; dans la deuxième, terminée en 784 , le compte à rebours est réduit à seize ans9. Dans les deux versions, Beatus conclut en reprenant le dernier paragraphe de la Chronique d'Isidore de Séville : la connaissance du temps qui reste est insondable pour l'homme ${ }^{10}$.

7 En dépit de cette conclusion parfaitement orthodoxe, aux yeux de certains historiens, le calcul de Beatus serait révélateur de son millénarisme, c'est-à-dire de son attente imminente de la fin des temps. Le calcul doit cependant être lu au regard de sa position au sein du Commentaire : il s'insère dans un long excursus sur les six âges du monde dont le point de départ est le commentaire du nombre des élus de l'Apocalypse, les 144000 ; les nombres mentionnés dans les Écritures ayant un sens et une valeur symboliques, Beatus s'appuie de manière classique sur la numérologie pour interpréter le nombre des élus et révéler la structure générale de l'histoire du salut ${ }^{11}$. Il en vient à montrer que le nombre six - soit la moitié de 12, la racine carrée de 144 qui associé au nombre 1000 forme le nombre des élus - y occupe une place centrale : il est le dénominateur commun d'une série de nombres clefs en rapport avec la résurrection ${ }^{12}$. Le nombre 6000 auquel aboutit le calcul de la date de la fin des temps est donc pour Beatus 
éminemment évocateur : il associe le chiffre 6 , symbole de la complétude de la création, et le nombre 1000, qui traduit la totalité et l'éternité, la perfection du dessein divin. Il ne faut, dès lors, pas comprendre le calcul selon la lettre, mais selon l'esprit. Beatus fait ici œuvre d'instructeur: les sciences du quadrivium, et plus particulièrement la numérologie, sont des outils qui permettent l'interprétation du texte biblique et donc l'accès à la compréhension du plan du salut dans une perspective eschatologique. Compter le temps et éprouver de l'intérêt pour l'arithmétique et l'arithmologie n'est pas seulement, comme l'a rappelé John Contreni, lié à la symbolique des nombres et à leur valeur allégorique. La science des nombres est, comme la science des lettres, un moyen «d'approcher le Créateur " "13. L'exactitude des mesures et des nombres reflète la droiture de l'action, de la pensée et de la parole ainsi que la perfection de la création. Beatus s'insère ainsi dans une tradition exégétique érudite dans laquelle l'eschatologie est à la fois un champ de la connaissance parmi d'autres et un horizon d'attente qui donne aux savoirs libéraux, propédeutiques de l'exégèse, une finalité.

On pourrait objecter que si cette lecture des computs est recevable dans le cadre de l'exégèse, leur copie dans d'autres contextes est susceptible d'autres interprétations. De fait, plusieurs manuscrits ibériques contiennent un calcul de l'âge du monde inséré dans des contextes variés (historiographie, liturgie, Bible), parfois accompagné d'un décompte des années qui restent à l'approche de la date estimée du sixième âge. Pour Juan Gil et Richard Landes qui les ont étudiés, ces computs seraient révélateurs d'une tension apocalyptique forte autour de l'an 800, puis 900 et enfin de l'an Mil ${ }^{14}$. Pourtant, il faut bien voir que si certains ont été mis à jour une fois la date dépassée, tous sont connus par des copies largement postérieures à la date prévue par le décompte; ils étaient très généralement copiés tels quels, sans actualisations ni modifications, mais souvent accompagnés de mises en garde parfaitement topiques sur l'inutilité de chercher à calculer la fin des temps ${ }^{15}$. Il faut, dès lors, sortir d'une conception utilitariste de ces calculs dont le succès ne s'est pas démenti une fois la date de la fin des temps dépassée : comme l'a montré Lars Hartman à partir des exemples judaïques, les contextes de lecture et de transmission des computs apocalyptiques révèlent un ensemble de fonctions qui dépassent largement leur seule dimension informative et calendaire ${ }^{16}$.

De fait, les calculs du temps qui reste jusqu'à la fin du sixième âge apparaissent dès l'époque wisigothique en appendice de deux types de textes principaux: les chroniques $^{17}$ et les computs pascaux ${ }^{18}$. Ils s'insèrent dans une tradition computistique longue, déjà en place alors que le calcul n'était pas lié à l'approche de l'échéance apocalyptique. Utilisées par les historiographes pour synchroniser les différents systèmes de datation en usage ou par les liturgistes pour calculer la date de Pâques, les dates des six âges offrent une approche chiffrée globale de la chronologie de l'histoire humaine ${ }^{19}$. La date de la fin des temps agit en ce sens comme repère temporel symbolique afin de se situer dans le plan du salut.

10 Au-delà de la date, les prophéties eschatologiques, canoniques comme apocryphes, constituent la première source de savoir sur la fin des temps. Dans l'Occident médiéval, elles n'ont pas été lues dans le cadre d'une attente angoissée de la fin des temps, mais bien comme supports de savoirs multiples. Comme l'a montré Anke Holdenried, la copie de l'Oracle de la Sibylle Tiburtine dans des recueils variés montre qu'elle répondait à des intérêts divers: curiosité pour l'Antiquité, compilation historiographique, christologie, etc. ${ }^{20}$. 
11 Dans la Péninsule, des textes décrivant la fin des temps ont été insérés dans des compilations dédiées à l'enseignement ou à la catéchèse, faisant ainsi l'objet d'un usage scolaire ou encore pastoral. Cette idée est notamment démontrée par le contexte de copie d'un petit traité andalou du IX siècle, l'Indiculus de aduentu Henoch et Eliae ${ }^{21}$. Ce texte constitue la plus ancienne synthèse sur l'Antichrist composée au haut Moyen Âge. Décrivant à partir de Daniel et de l'Apocalypse les signes qui précéderont la venue de l'Antichrist, son règne, puis sa défaite, il est constitué d'extraits de plusieurs ouvrages, dont le Sur Daniel de Jérôme et les Commentaires sur l'Apocalypse de Victorin de Poetovio, dans la version revue par Jérôme, et de Beatus de Liébana ${ }^{22}$. Le texte suit une construction logique : en partant de la conversion des juifs à la fin des temps grâce à la prédication du prophète Élie, l'Indiculus décrit comment l'Antichrist tuera les deux témoins annoncés par l'Apocalypse (11), Hénoch et Élie, avant de décrire comment il séduira, par la tromperie, le peuple et notamment les juifs, qui le recevront comme leur Messie. Le texte rassemble plusieurs arguments patristiques sur l'origine juive de l'Antichrist, condensant diverses sentences de tonalité antijudaïque dans une tradition bien établie depuis l'époque wisigothique.

12 Le plus ancien témoin du texte est un manuscrit composite, assemblé et complété au $\mathrm{IX}^{\mathrm{e}}$ siècle en al-Andalus ${ }^{23}$. Il rassemble des extraits d'œuvres choisies autour de deux textes principaux, le De natura rerum et la Chronique d'Isidore de Séville. Le manuscrit contient des extraits des Étymologies, de divers opuscules géographiques et historiques, des Instructions d'Eucher de Lyon, ou le sermon Sur la paix d'Ambroise. L'Indiculus a été ajouté sur des folios palimpsestes lors de la recomposition du manuscrit. Ses caractéristiques codicologiques - un livre de petite taille, maniable, comportant de nombreuses traces d'usage - ainsi que la composition du recueil sont compatibles avec un manuscrit scolaire; il s'agit vraisemblablement d'un manuel. L'ajout de l'Indiculus complète le discours historique, en ajoutant un compendium de lecture aisée sur la fin des temps à une compilation de textes divers, historiques, géographiques et théologiques.

Le deuxième exemplaire conservé de l'Indiculus figure dans un manuscrit composite du $\mathrm{X}^{\mathrm{e}}-\mathrm{XI}^{\mathrm{e}}$ siècle - CÓRDOBA, Archivo Capitular, $123^{24}$. Le premier secteur du manuscrit contient les œuvres presque complètes d'un auteur andalou du $\mathrm{IX}^{\mathrm{e}}$ siècle, Alvare de Cordoue (fol. 1-164); le second (fol.165-207), dans lequel fut copié l'Indiculus, comprend des Quaestiones de trinitate, un pénitentiel, un De genealogiis, un sermon d'Augustin sur l'arbre de la connaissance, et enfin un texte sur l'origine du Credo. Ces textes sont des ouvrages généraux, voire introductifs, de la doctrine chrétienne; l'insertion de l'Indiculus dans cet ensemble confirme son caractère de compendium accessible sur les fins dernières: il pourrait avoir été ici utilisé afin d'introduire les connaissances élémentaires de la doctrine eschatologique chrétienne dans un contexte catéchétique ou pastoral. On peut envisager que la dimension antijudaïque du traité ait servi à renforcer la croyance dans la foi chrétienne dans un contexte de polémique religieuse exacerbé après la conquête islamique : les chrétiens d'al-Andalus vivent en effet sous le statut de la dhimma, au même titre que les juifs, ce qui leur permet de conserver leurs coutumes et leur religion en échange du paiement d'un impôt. Les communautés chrétiennes sont confrontées à la tentation de l'apostasie au profit de l'islam et à une recrudescence de la polémique judéo-chrétienne ${ }^{25}$.

14 Un contexte de transmission similaire éclaire les fonctions de la seule révélation sur la fin des temps composée dans la Péninsule au haut Moyen Âge: sous la forme d'un 
dialogue entre le roi Aristote et l'évêque Alexandre ${ }^{26}$, l'Interrogatio de nobissimo décrit les signes annonçant la fin du monde, la venue de l'Antichrist, sa mort et enfin la résurrection des corps et le Jugement ${ }^{27}$. La liste des signes emprunte largement aux traditions apocryphes; elle comporte des signes naturels ou célestes, mais la majorité sont sociétaux ou moraux - multiplication de l'impiété, des guerres, des vols, inversion des hiérarchies sociales, etc. La description de l'Antichrist repose également largement sur les traditions antérieures, néanmoins quelques éléments y sont originaux, tels que la mention du fait qu'il marchera sur les mers. La prophétie n'est transmise que par un témoin composite $\mathrm{du} \mathrm{Ix}^{\mathrm{e}}$ siècle d'origine inconnue, peut-être navarro-pyrénéenne, entré à une date incertaine dans le fond de la bibliothèque de San Millán de la Cogolla, où il a été abondamment glosé jusqu'à la fin $\mathrm{du} \mathrm{XI}^{\mathrm{e}}$ siècle $^{28}$. L'Interrogatio a été copiée dans le secteur $C$ du manuscrit (fol. $50 v^{\circ}-96 v^{\circ}$ ), au sein d'un ensemble de sermons desquels rien ne le distingue d'un point de vue matériel ${ }^{29}$. Les sermons ne sont pas destinés à une célébration particulière du calendrier liturgique, mais sont de lecture quotidienne; tous invitent à la pénitence et entretiennent la tension eschatologique. L'ajout d'une prophétie sur la fin des temps à cet ensemble a ainsi sans doute été envisagé comme un moyen supplémentaire d'inciter à la pénitence par une description marquante de la fin des temps.

Sortis de leur contexte, ces textes sur les signes de la fin du monde et la venue de l'Antichrist pourraient témoigner d'un intérêt peut-être teinté d'inquiétudes ou d'attentes apocalyptiques. Leur transmission au sein de manuscrits, qui ont vraisemblablement servi de manuels scolaires, catéchétiques ou de supports à la méditation, indique qu'ils constituaient l'un des biais de transmission du discours savant sur la fin des temps élaboré par les lettrés, ici rassemblé sous forme de compendiums. De composition simple, reprenant le scénario eschatologique classique, ces textes ont été écrits afin de transmettre un enseignement élémentaire sur les fins dernières, sans urgence apocalyptique.

\section{L'exégèse des prophéties eschatologiques : une technique intellectuelle et spirituelle entre érudition et méditation}

Le discours savant sur les fins dernières prend plus particulièrement la forme de l'exégèse à travers commentaires, traités ou florilèges. En dépit d'une production originale, apparemment limitée au seul Commentaire de Beatus, l'exégèse sur les livres eschatologiques de la Bible a connu un renouveau dans le haut Moyen Âge ibérique à travers des chaînes exégétiques ou florilèges anonymes et des corpus d'annotations. Un manuscrit copié en al-Andalus au début du Ix siècle - MADRID, Real Academia de la Historia, $80^{30}$ - contient notamment une chaîne sur l'Apocalypse utilisant comme guide principal le commentaire de Victorin ${ }^{31}$, complété de trois homélies sur l'Apocalypse tirées de l'homéliaire de Smaragde de Saint-Mihiel ${ }^{32}$. La chaîne figure dans un manuscrit contenant d'autres commentaires bibliques, qui se présente comme une compilation exégétique centrée sur quatre livres d'interprétation difficile de la Bible le Cantique des Cantiques, les Évangiles de Matthieu et Jean, l'Apocalypse -, pour lesquels la lecture allégorique est centrale. Le compilateur a vraisemblablement ici 
voulu compléter le Commentaire relativement succinct de Victorin et fournir un guide de lecture allégorique de l'Apocalypse.

Deux autres florilèges copiés au $\mathrm{IX}^{\mathrm{e}}$ siècle en al-Andalus révèlent des fonctions différentes : à partir du Commentaire sur Matthieu de Jérôme, un compilateur anonyme a réalisé un travail inédit de sélection d'extraits, réordonnés autour de la thématique de la fin des temps, peut-être dans le but de composer un nouveau traité ${ }^{33}$. Un autre a rassemblé, dans un but plutôt polémique, des passages du Commentaire sur Daniel de Jérôme et du De fide catholica contra Iudaeos d'Isidore de Séville autour de la prophétie des soixante-dix semaines de Daniel 9 et la venue de l'Antichrist ${ }^{34}$. Deux bibles des IX et $\mathrm{x}^{\mathrm{e}}$ siècles contiennent des corpus d'annotations fournis sur les Prophètes et

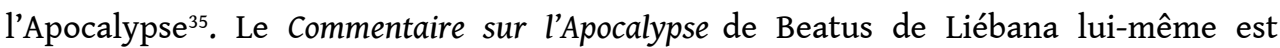
devenu le support privilégié d'un enseignement sur des thèmes aussi divers que le temps, le paradis, ou l'Antichrist. Des extraits de l'ouvrage ont en effet été utilisés afin de servir de base à plusieurs compositions originales, notamment l'Indiculus de aduentu Henoch et Eliae adque Antichristi mentionné précédemment, un florilège de textes centrés sur la symbolique des nombres de l'histoire du salut, de la création à la fin des temps dont un texte intitulé le De fine mundi ${ }^{36}$, ou encore des Questions-réponses sur la Jérusalem céleste copiées à la fin du $\mathrm{x}^{\mathrm{e}}$ siècle dans un monastère de Castille ${ }^{37}$.

On peut s'interroger sur cette recrudescence de petits traités, florilèges et commentaires consacrés à l'eschatologie dans le haut Moyen Âge ibérique ; la doctrine sur les fins dernières est après tout fixée dans ses grandes lignes depuis Augustin et Jérôme, une synthèse en a même été réalisée à la fin du viI ${ }^{\mathrm{e}}$ siècle par Julien de Tolède, le Prognosticum futuri saeculi. Sommet des sciences, l'exégèse est, comme l'écrivait Jean Leclercq, la salutaris scientia ${ }^{38}$, c'est-à-dire qu'elle révèle le message adressé par Dieu à travers l'Écriture pour le salut, dans le présent, et en vue de la fin des temps ${ }^{39}$. Elle est également une technique intellectuelle qui répond à un ensemble de $\operatorname{lois}^{40}$. Seuls les moines les plus avancés s'y consacrent. Ce renouveau atteste de la volonté de comprendre et de transmettre, voire de compléter l'héritage des Pères; il est également significatif du fait que l'eschatologie est au Moyen Âge, dans la Péninsule comme plus largement en Occident, un champ de recherche qui n'est pas figé.

De fait, les lettrés rassemblent, confrontent, hiérarchisent ou juxtaposent un ensemble de connaissances sur la fin des temps acquises par l'étude et l'approfondissement des Écritures, et ces données sont parfois contradictoires, à l'image du décompte de l'âge du monde associé au refus augustinien du calcul du temps qui reste chez Beatus ou chez l'auteur anonyme du De fine mundi. Pour Richard Landes, ce paradoxe ne peut s'expliquer que comme les traces de l'existence de deux discours contradictoires, l'un écrit et orthodoxe, l'autre oral et millénariste ${ }^{41}$. Ces deux positions ne relèvent pourtant pas nécessairement d'une contradiction irrésoluble aux yeux des médiévaux ${ }^{42}$. Comme l'a montré Henri de Lubac, les exégètes conciliaient les apparents désaccords des Pères en usant d'un ensemble de formules topiques, elles-mêmes inspirées des écrits patristiques destinés à résoudre les contradictions des Écritures ${ }^{43}$. La juxtaposition de données opposées dans l'élaboration d'un discours cohérent n'était pas perçue comme problématique dans la mesure où le texte était conçu comme objet d'une herméneutique qui faisait apparaître plusieurs niveaux de lectures successifs ${ }^{44}$; la pratique d'un savoir cumulatif ${ }^{45}$, en particulier dans l'exégèse, fournit le cadre de cette lecture érudite. Autrement dit, confronté à une diversité d'opinions, le lettré du haut Moyen Âge les répertorie, les étudie successivement et les interprète en conformité 
avec une vérité, celle de la foi. Le cas des péricopes bibliques sur la connaissance de la date et de l'heure de la fin des temps est révélateur de cette méthode.

Alors que plusieurs passages des Écritures affirment la proximité du retour du Christ, voire annoncent la multiplication des signes qui le précéderont immédiatement ${ }^{46}$, d'autres assurent que la fin des temps se produira de manière inopinée et surprendra les croyants de telle sorte que ceux-ci doivent en permanence se tenir prêts ${ }^{47}$. Les exégètes, à l'image de Beatus, interprètent généralement ces passages en soulignant que la connaissance de la date de la fin du monde est inaccessible à l'homme, citant la réponse du Christ à ses apôtres qui l'interrogent à ce sujet: Quant à la date de ce jour, et à l'heure, personne ne les connaît, ni les anges des cieux, ni le Fils, personne que le Père, seul ${ }^{48}$. Lue de manière littérale, la citation semble remettre en question la toute-puissance du Christ, son égalité avec le Père et donc les fondements de la foi chrétienne. Elle apparaît ainsi de manière récurrente dans les attaques polémiques contre les chrétiens depuis Jérôme, et notamment dans l'arianisme. En évoquant des contradicteurs peut-être fictifs, Beatus leur répond par une interprétation du passage ne laissant pas de place au doute: «il nous faut en vérité comprendre ceci non selon la lettre, mais selon l'idée » [...] «il est évident que le Fils savait, mais qu'il ne voulait pas l'indiquer à ses disciples afin que toujours attendant la mort et sans certitude, ils n'aient pas d'espoir dans le siècle ${ }^{49}$ ». Au milieu du Ix ${ }^{e}$ siècle, à Cordoue, l'abbé Speraindeo répond dans un traité composé contre l'islam, mais destiné aux chrétiens, à une interrogation fictive qui confronte ces deux thèses en citant longuement Matthieu 24, 36 et Matthieu 24, $3-30^{50}$. Sa réponse, semblable à celle de Beatus, fait le parallèle avec d'autres possibles incohérences dans les Écritures, par exemple le fait que Dieu demande à Adam où il se cache, ou à Caïn, où se trouve Abel, etc., et invite l'obstiné à réfléchir là-dessus.

21 Autrement dit, en dépit d'un phénomène au long cours d'homogénéisation et de fixation progressive du discours sur la fin des temps, la composition de textes sur l'eschatologie au haut Moyen Âge préserve des représentations contradictoires. Ces textes forment un discours pluriel qui ne cherche pas à imposer l'unicité d'une interprétation des mystères des Écritures, mais au contraire à en préserver la variété des niveaux de lectures, ceux-ci étant en eux-mêmes diversement accessibles aux chrétiens.

Le renouveau de l'exégèse touchant à l'eschatologie dans la Péninsule est révélateur de sa place centrale dans l'enseignement des moines. L'élaboration d'un savoir sur les fins dernières n'est pas la seule finalité du travail de l'exégète qui accède, par la lecture et la méditation des Écritures, à la contemplation de Dieu. En ce sens, l'exégèse des prophéties sur la fin des temps est une technique tout autant spirituelle qu'intellectuelle, support d'une activité méditative. Il n'y a rien ici d'original : Jean Leclercq a bien su restituer comment la littérature monastique est en tension vers un accomplissement, la contemplation de Dieu à la fin des temps. Au moine qui désire atteindre cet accomplissement, la lectio diuina fournit les clefs de la méditation ${ }^{51}$; Mary Carruthers, à travers ses travaux sur la mémoire, a mis en évidence comment celle-ci, entendue en un sens très large, est un rouage essentiel de la pensée médiévale et nourrit la méditation ${ }^{52}$; Brian Stock, dans ses travaux sur la lectio diuina, montrait qu'elle était envisagée comme un moyen d'atteindre un état d'esprit contemplatif ${ }^{53}$. Des traces de cette activité peuvent être observées dans les marges de textes eschatologiques. Ainsi, à la fin d'un manuscrit ibérique des Moralia de Grégoire le Grand, une main inconnue a inscrit "pour désirer le royaume, tu as perdu le sens; 
ouvre le sens et tu acquerras le royaume $»^{54}$. Bien que cryptique, la note semble faire référence au désir du royaume céleste auquel la lecture, par l'ouverture du sens - ou de la compréhension - permettrait d'accéder et dans laquelle il est possible de se perdre. La recherche du «sens » peut ainsi faire référence au mécanisme mnémotechnique de la pensée, qui en associant mot, idée et symbole, fait advenir un sens cachés5. De fait, les moines atteignaient la contemplation de Dieu par la manducation des Écritures et des Pères : en utilisant le pouvoir de l'imagination et la mnémotechnie, la lecture provoque une émotion ou une réaction esthétique chez le lecteur, qui mène à la méditation par la puissance évocatrice des images textuelles ou visuelles ${ }^{56}$. Dans la littérature monastique, notamment la Vie de saint Antoine ou L'échelle du paradis de Jean Climaque, et jusqu'au Commentaire sur l'Apocalypse de Beatus, les moines sont invités à méditer sur le verset de l'Ecclésiaste $(7,36 / 40)$ : «Rappelez-vous vos fins dernières, et vous ne pécherez jamais ${ }^{57}$.» Comme le soulignait Mary Carruthers, dans le langage monastique courant «on se souvient des fins dernières »: la prière vétérotestamentaire « souviens-toi de Jérusalem » n'est pas une injonction à se tourner vers le passé, mais à agir en vue du futur eschatologique ${ }^{58}$. La memoria est ce qui permet de percevoir les choses futures par leur ressemblance avec les choses passées ${ }^{59}$.

Dans la péninsule Ibérique, les Beatus ont vraisemblablement occupé, à côté des Bibles et des homéliaires, une place prépondérante comme supports de la lectio diuina ${ }^{60}$. Dans son prologue, Beatus établit un lien étroit entre la lecture et la mémoire : les prophéties ont été reprises et expliquées à travers de nombreux ouvrages et par de nombreux pères, mais «quoique toutes ces choses soient connues de ceux qui parcourent longuement les Écritures, il est plus facile de se les remémorer quand on les lit dans une parole brève ${ }^{61}$ ». Plusieurs travaux ont mis en évidence comment le Commentaire a été conçu afin d'inviter le lecteur à s'immerger dans le texte et l'image et, ainsi, à s'élever vers la contemplation ${ }^{62}$.

D'après Brian Stock, si la lectio diuina ne se pratique pas d'ordinaire en silence, elle est considérée comme menant à la méditation silencieuse ${ }^{63}$. Beatus évoque en ce sens le silence dans le ciel d'Apocalypse 8, $1:$ :"Il y eut dans le ciel un silence comme d'une demi-heure", c'est-à-dire, les serviteurs de Dieu, qui s'abstiennent de toute activité du siècle et commencent dans la contemplation à goûter la vie éternelle ${ }^{64}$. Si l'activité contemplative est ici clairement réservée aux moines et opposée, suivant le modèle grégorien, au travail manuel, dans d'autres passages de son Commentaire, Beatus décrivait l'activité de lecture et de méditation des Écritures plus largement comme ce qui définit l'homme et le différencie de l'animal, comme elle distingue l'Église de la Synagogue ${ }^{65}$. Dans les deux cas, il lie cette activité à la capacité de l'homme à percevoir le passé, le présent et le futur: c'est bien par la compréhension des Écritures que l'homme peut atteindre cette vision de l'ensemble des temps qui est synonyme de la contemplation de Dieu. La méditation des fins dernières embrasse en réalité la compréhension du dessein divin et de l'histoire du salut, récapitulée dans le cycle liturgique; son explication est recherchée par les docteurs à travers l'exégèse afin d'être offerte au plus grand nombre par la prédication. 


\section{Conclusion} et de contemplation nourries par une approche érudite. L'analyse des florilèges ou traités eschatologiques composés dans le haut Moyen Âge ibérique souligne qu'en dépit d'une production exégétique trop souvent réduite au seul Commentaire de Beatus, les lettrés ont élaboré de nouveaux textes dans des buts polémiques, scolaires ou encore méditatifs. Maîtrisant les différentes techniques de l'exégèse, les auteurs anonymes ont abordé l'eschatologie comme un champ d'investigation toujours actif, s'efforçant notamment de résoudre les contradictions dans les Écritures et entre les Pères. Le commentaire de la Bible et la lecture des Pères - principaux supports de l'élaboration d'un savoir croyant sur l'eschatologie - sont ainsi à l'intersection même de l'édification d'un discours érudit et d'une pratique spirituelle et religieuse.

Reçu : 25 juillet 2020 - Accepté : 20 novembre 2020

\section{NOTES}

1. La liste quasi exhaustive de ces textes a été établie par les publications de M. C. DÍAZ y DÍAZ, Index scriptorum latinorum Medii Aevi hispanorum, Salamanque, 1958, 2 vol. et J. C. MARTíN-IGLESIAS, Sources latines de l'Espagne tardo-antique et médiévale, $V^{e}-\mathrm{XIV}$ siècles : répertoire bibliographique, Paris, 2010.

2. Pour une première approche de l'importante bibliographie sur ce commentaire, voir: R. GRYSON, "Introduction », in BEATUS DE LIÉBANA, Tractatus de Apocalipsin, éd. R. GRYSON, Turnhout, 2012 (Corpus Christianorum, Series Latina, 107B-C); P. HENRIET, «Compte rendu. Beatus de Liébana, Tractatus de Apocalipsin, cura et studio Roger Gryson...", Revue Mabillon, 86 (2014), p. 373-376; et sur les manuscrits : J. W. WiLliams, The Illustrated Beatus : A Corpus of the Illustrations of the Commentary on the Apocalypse, Londres, 1994, 5 vol.; ID., Visions of the End in Medieval Spain, Amsterdam, 2016. 
3. J. GIL FERNÁNDEZ, "Judíos y cristianos en Hispania (s. VIII y IX)", in Hispania sacra. Revista española de historia eclesiástica, 31 (1978), p. 9-88, ici p. 11.

4. Ce débat a notamment opposé les tenants de «l'école des terreurs » dont, en premier lieu, R. Landes ou J. Fried à S. Gouguenheim, voir: R. LANDES, "Lest the Millennium be Fulfilled: Apocalyptic Expectations and the Pattern of Western Chronography, 100-800 CE », in W. VERBEKE, D. VERHELST et A. WELKENHUYSEN (dir.), The Use and Abuse of Eschatology in the Middle Ages, Louvain, 1988, p.137-211; ID., «Millenarismus absconditus: l'historiographie augustinienne et le millénarisme du haut Moyen Âge jusqu'à l'an mil », in Le Moyen Âge, 98 (1992), p. 355-377 ; ID., "Sur les traces du Millennium : la "Via Negativa" », Le Moyen Âge, 99 (1993), p. 5-26 ; ID., " The Fear of an Apocalyptic Year 1000. Augustinian Historiography, Medieval and Modern ", Speculum, 75 (2000), p. 97-145 ; J. FRIED, «Endzeiterwartung um die Jahrtausendwende », Deutsches Archiv für Erforschung des Mittelalters, 45 (1989), p. 381-473 ; J. FRIED, Les fruits de l'Apocalypse: origines de la pensée scientifique moderne au Moyen Âge, trad. fr. D. Modigliani, Paris, 2004 ; S. GoUguenheIM, «L'histoire d'un mythe : l'invention des terreurs de l'an mil. Étude et critique historiographique d'Abbon de Fleury à Richard Landes ", ALMA, Bulletin du Cange, 57 (1999), p. 112-190 ; ID., Les fausses terreurs de l'an mil : attente de la fin des temps ou approfondissement de la foi ?, Paris, 1999.

5. J. FONTAINE, «Fuentes y tradiciones paleocristianas en el método espiritual de Beato ", in Actas del Simposio para el estudio de los códices del «Comentario al Apocalipsis» de Beato de Liébana, Madrid, 1978, t. 1, p. 75-103 ; A. A. NASCIMENTO, « O "Comentário ao Apocalipse” de Beato de Liébana : entre gramática e escatologia», Euphrosyne, 28 (2000), p.129-156; contra R. Landes, «Lest the Millennium... ", op. cit.

6. J. GIL FERNÁNDEZ, «Los terrores del año 800 ", in Actas del simposio para el estudio..., ibid., t. 1, p. 224-226 ; R. LANDES, "Lest the millenium... », op. cit., p. 192-194 ; A. RUCQUOI, " Mesianismo y milenarismo en la España medieval », Medievalismo, 6 (1996), p. 9-31, ici p. 12-13 et 19; EAD., «El fin del milenarismo en la España de los siglos X y XI », in J. DE LA IGLESIA DUARTE (dir.), Milenarismos y milenaristas en la Europa Medieval. IX Semana de Estudios Medievales, Nájera, 1998, Logroño, 1999, p. 281-304, ici p. 284 ; EAD., « Medida y fin de los tiempos. Mesianismo y milenarismo en la Edad Media ", in Á. VACA LORENZo (dir.), En pos del tercer milenio. Apocalíptica, mesianismo, milenarismo e historia. Undécimas Jornadas de Estudios Históricos organizadas por el Departamento de Historia Medieval, Moderna y Contemporánea, Salamanque, 2000, p. 13-41, ici p. 21 ; J. FLORI, L'Islam et la fin des temps : l'interprétation prophétique des invasions musulmanes dans la chrétienté médiévale, Paris, 2007, p. 150-155.

7. Sur cette tradition voir: J. DANIÉLOU, «La typologie millénariste de la semaine dans le Christianisme primitif », Vigiliae christianae, 2 (1948), p. 1-16; A. LUNEAU, L'histoire du salut chez les Pères de l'Église : la doctrine des âges du monde, Paris, 1964 ; P. ARCHAMBAUlt, « The Ages of Man and the Ages of the World: A study of two Traditions ", Revue d'études augustiniennes, 12/2 (1966), p. 193-228 ; B. GUENÉE, Histoire et culture historique dans l'Occident médiéval, Paris, 1980, p. 147-154 ; C. CAROZZI, Apocalypse et salut dans le christianisme ancien et médiéval, Paris, 1999, p. 35-61; É. MÉGIER, "Le temps des âges du monde, de saint Augustin à Hugues de Fleury (en passant par Isidore de Séville, Bède le Vénérable, Adon de Vienne et Fréculphe de Lisieux) », in P. BOURGAIN, J.-Y. TILLIETTE et J. M. ZIOLKOWSKI (dir.), Le sens du temps: actes du VII congrès du Comité international de latin médiéval [The Sense of Time: proceedings of the 7th Congress of the International medieval latin commitee], Genève, 2017, p. 581-600 ; sur son adaptation dans la péninsule Ibérique voir : N. ROTH, " "Seis edades durará el mundo". Temas de la polémica judía española », La Ciudad de Dios, 149/1 (1986), p. 45-65.

8. "Quotquot enim diebus hic factus est mundus, tot et millenia annis consummatur. Et propter hoc ait Scriptura Geneseos: "Et consummata sunt caelum et terra et omnis ornatum eorum. Et consummauit Deus die sexto omnia opera sua quae fecit, et requieuit in die septimo ob omnibus operibus suis quae fecit". Hoc autem est et ante factorum narratio quemadmodum facta sunt et futurorum prophetia. Si enim "dies 
Domini quasi milla anni", in sex autem diebus consummata sunt quae facta sunt, manifestum est quoniam consummatio ipsorum sextus millesimus annus est. Car autant de jours a comporté la création du monde, autant de millénaires comprendra sa durée totale. C'est pourquoi le livre de la Genèse a dit : "Ainsi furent achevés le ciel et la terre et toute leur parure. Dieu acheva le sixième jour les œuvres qu'il fit, et Dieu se reposa le septième jour de toutes les œuvres qu'il avait faites" (Gen. 2, 12). Ceci est à la fois un récit du passé tel qu'il se déroula et une prophétie de l'avenir : en effet, si "un jour du Seigneur est comme mille ans" (II Pet. 3, 8, cf. Ps. 89, 4) et si la création a été achevée en six jours, il est clair que la consommation des choses aura lieu la six millième année ", IRÉNÉE DE Lyon, Contre les hérésies, éd. A. ROUSSEAU, L. DOUTRELEAU, et C. MERCIER, Paris, 1969, Liv. V, § 28, 3 , t. 2, p. 358-359 (Sources chrétiennes, 153).

9. Voir sur ce point R. GRYSON, «Introduction », in BEATUS, Tractatus, t. 1, p. XLII-XLVI.

10. BEATUS, Tractatus, IV, 5 , § 13-17, éd. R. GRYSON, t. 2, p. 516-518.

11. BEATUS, Tractatus, IV, 5, § 44-68 puis $\S 79$ et 105, éd. R. GRYSON, t. 2, p. 526-543.

12. R. GRYSON, "Introduction", ibid., p. XLI-XLII; G. BOSSEMAN, "Beatus of Liebana and the spiritualized understanting of Apocalypse in Medieval Iberia », in H.-C. LEHNER (dir.), Endzeiten End(s) of Time, Leyde, à paraître.

13. J. J. CONTRENI, « Counting, Calendars, and Cosmology : Numeracy in the Early Middle Ages », in J. J. CONTRENI et S. CASCIANI (dir.), Word, Image, Number: Communication in the Middle Ages, Florence, 2002, p. 43-83, repris dans J. J. CONTRENI, Learning and Culture in Carolingian Europe : Letters, Numbers, Exegesis, and Manuscripts, Farnham, 2011, III, p. 43-83, ici p. 52. Sur la place de l'arithmologie dans l'enseignement, voir: J. J. CONTRENI, "The Pursuit of Knowledge in Carolingian Europe ", in R. E. SUluIVAN (dir.), "The Gentle Voices of Teachers": Aspects of Learning in the Carolingian Age, Colombus, 1995, p. 106-141, ici p. 124. Sur la valeur allégorique des nombres, voir aussi : H. MEYER, Die Zahlenallegorese im Mittelalter: Methode und Gebrauch, Munich, 1975 ; H. MEYER et R. SUNTRUP, Lexikon der mittelalterlichen Zahlenbedeutungen, Munich, 1987.

14. R. LANDES, "Lest the Millenium...», op. cit., et pour les sources ibériques : J. GIL FERNÁNDEZ, «Judíos y cristianos... », op. cit., p. 71-72, 75 ; ID., « Textos olvidados del Códice de Roda », Habis, 2 (1971), p. 165-178, ici p. 171-173 ; A. RUCQUOI, « Mesianismo y milenarismo... », op. cit., p. 12.

15. Contrairement à ce qu'écrit J. Fried, il n'est pas rare que de tels computs aient été transmis déjà dépassés, cf. J. FRIED, Les fruits de l'Apocalypse..., op. cit., p. 51.

16. L. HARTMAN, "The Functions of some so-called Apocalyptic Timetables", New Testament Studies, 22/1 (1975), p. 1-14.

17. R. LANDES, «Lest the Millennium... », op. cit., p. 168-174 ; J. T. PALMER, «Calculating Time and the End of Time in the Carolingian World, c. 740-820», The English Historical Review, 126 (2011), p. 1307-1331; ID., "The Ordering of Time», in V. WIESER, C. ZOLLES, C. FEIK, M. zOLLES et L. SCHLÖNDORFF (dir.), Abendländische Apokalyptik. Kompendium zur Genealogie der Endzeit, Berlin, 2012, p. 605-618.

18. Voir notamment les contextes de transmission de l'Ordo annorum mundi, un petit traité récapitulatif des six âges avec un calcul des années écoulées depuis la création: PS-JULIEN DE TOLÈDE, Ordo annorum mundi, éd. J. C. MARTÍN-IGLESIAS, in JULIEN DE TOLÈDE, Opera, Pars 2, Turnhout, 2014, t. 1, p. 260-279 et t. 2, p. 689-704 (Corpus Christianorum, Series Latina, 115A-B). Sur le contexte liturgique, voir: J. T. PALMER, «Computus after the Paschal Controversy of AD 740 », in I. WARNTJES et D. ó CRÓINÍN (dir.), The Easter Controversy of Late Antiquity and the Early Middle Ages : its manuscripts, texts, and tables, Turnhout, 2011, p. 213-241 ; I. WARNTJES, « The Final Countdown and the Reform of the Liturgical Calendar in the Early Middle Ages ", in M. GABRIELE et J. T. PALMER (dir.), Apocalypse and Reform from Late Antiquity to the Middle Ages, Londres, 2019, p. 51-75.

19. H. INGLEBERT, Interpretatio christiana: les mutations des savoirs (cosmographie, géographie, ethnographie, histoire) dans l'Antiquité chrétienne, 30-630 après J.-C, Paris, 2001, p. 380. 
20. A. HOLDENRIED, The Sibyl and her Scribes: Manuscripts and Interpretation of the Latin Sibylla Tiburtina c. 1050-1500, Aldershot/Burlington, 2006.

21. Indiculus de aduentu, in Scriptores Muzarabici saeculi VIII-XI, éd. J. GIL FERNÁNDEZ, Turnhout, 2020, t. 1, p. 348-358 (Corpus Christianorum, Continuatio Mediaevalis, 65A); datation et origine proposée par M.C. DíAZ Y DÍAZ, «El pseudoieronimiano "De adventu Henoch" ", Überlieferungsgeschichtliche Untersuchungen, 125 (1981), p.141-148, et M. DULAEY, "Victorin de Poetovio est-il l'auteur de l'opuscule sur l'Antéchrist publié par A. C. Vega ? ", Rivista di storia e letteratura religiosa, 21 (1985), p. 258-261.

22. G. BosSEMAN, « Circulation et usages de l'exégèse dans la péninsule Ibérique au IX ${ }^{e}$ siècle : étude des sources de l'Indiculus de adventu Enoch et Eliae adque Antichristi», Mélanges de la Casa de Velázquez, 49/1 (2019), p. 41-60.

23. M. C. DíAZ Y DíAZ, Códices visigóticos en la monarquía leonesa, León, 1983, p. 17-52 ; ID., Manuscritos visigóticos del sur de la península : ensayo de distribución regional, Séville, 1995, p. 64-69.

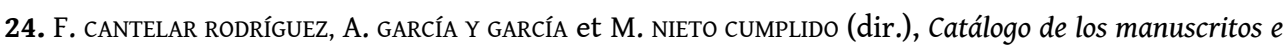
incunables de la catedral de Córdoba, Salamanque, 1976, p.211-214; M. C. DÍAZ Y DÍAZ, Códices visigóticos..., ibid., p. 370-374.

25. Sur les communautés andalouses, voir C. AILLET, Les Mozarabes: christianisme, islamisation et arabisation en péninsule Ibérique (IX ${ }^{e}-\mathrm{XII}{ }^{e}$ siècle), Madrid, 2010.

26. Sur le choix de ces personnages, bien connus dans l'eschatologie chrétienne, voir: J. GuAdalajARA medina, El Anticristo en la España medieval, Madrid, 2004, p. 44-46; M. A. SÁNCHEZ SÁNCHEZ, «Predicación, gramática y lectura : glosas a las glosas de San Millán y de Silos », in ID., La primitiva predicación hispánica medieval : tres estudios, Salamanque, 2000, p. 165-220, ici p. 168-172 ; C. GAULLIER-BOUGASSAS, «L'historiographie médiévale d'Alexandre : héritages, renouvellements et débats ", in EAD. (dir.), L'historiographie médiévale d'Alexandre le Grand, Turnhout, 2011, p. 5-36, et pour la péninsule Ibérique : H. de CARLOS VILLAMARÍN, "L'Alexandre historique et l'opposition de l'Occident et de l'Orient: les "Revelationes" du Pseudo-Méthode dans l'historiographie hispanique ", Ibid., p. 271-282.

27. MADRID, Real Academia de la Historia, 60 , fol. $64 \mathrm{r}^{\circ}-67 \mathrm{v}^{\circ}$.

28. Sur ce manuscrit, voir E. RUIZ GARCíA, Catálogo..., op. cit., p. 327-331. La présence de gloses, dont certaines en castillant datant $\mathrm{du} \mathrm{XI}^{\mathrm{e}}$ siècle sont les premières connues en cette langue vernaculaire, explique que le manuscrit ait fait l'objet de plusieurs éditions et études : M. C. DÍAZ Y DíAZ, Las primeras glosas hispánicas, Barcelone, 1978; S. GARCíA LARRAGUETA (éd.), Las Glosas emilianenses, Logroño, 1984 ; C. HERNÁNDEZ ALONSO (éd.), Las glosas emilianenses y silenses : ed. crítica y facsímil, Burgos, 1993 ; H. J. wolf (éd.), Las glosas Emilianenses, Séville, 1996. Nouvelle édition à paraître : éd. J. C. MARTín-IGLESIAS, in C. GARCía TURZA (dir.), Los textos latinos de base de las Glosas Emilianenses y Silenses : presentación, transcripción y traducción.

29. Les sermons ont été partiellement identifiés par Dom Férotin: M. FÉROTIN, A. WARD et C. JOHNSON (éd.), Le Liber mozarabicus sacramentorum et les manuscrits mozarabes, Rome, 1995, col. 899. Voir aussi : A. FRANQUESA, «El códice emilianense 60 y sus piezas litúrgicas ", Hispania Sacra, 12/24 (1959), p. 423-444 ; M. A. SÁNCHEZ SÁNCHEZ, « Predicación, gramática y lectura... », op. cit., p. 165-220.

30. MADRID, Real Academia de la Historia, 80 , fol. $142 \mathrm{r}^{\circ}-148 \mathrm{v}^{\circ}$; sur le manuscrit, voir : E. RUIZ GARCíA, Catálogo..., op. cit., p. 413-422.

31. R. GRYSON, « Introduction », in VICTORIN, Explanatio in apocalypsin, p. 41-44 et 84-88.

32. SMARAGDE DE SAINT-MIHIEL, Collectiones in espistulas et evangelia quae per circulum anni leguntur (ou Expositio libri comitis), éd. PL, 102, col. 9-593A, ici cap. I, col. 475C-477B, cap. IV, col. 331C-339A et cap. XIV, col. 49A-50A. Les extraits avaient été identifiés par F. RÄDLE, Studien zu Smaragd von SaintMihiel, München, 1974, p. 123, n. 81. Sur Smaragde, voir : J. LECLERCQ, « Smaragde et la grammaire 
chrétienne », Revue du Moyen Âge latin, 4 (1948), p. 15-22 ; A. DUBREUCQ, « Smaragde de Saint-Mihiel et son temps: enseignement et bibliothèques à l'époque carolingienne ", Mélanges de la Bibliothèque de la Sorbonne, 7 (1986), p. 7-36; P. RICHÉ, "Les réfugiés wisigoths dans le monde carolingien », in J. FONTAINE et C. PELLISTRANDI (dir.), L'Europe héritière de l'Espagne wisigothique, Madrid, 1992, p. 177-183, ici p. 181-182.

33. LEÓN, Archivo Catedral, 22, fol. 17-30.

34. MADRID, Biblioteca Nacional, 10018, fol. 181-189 ; texte partiellement édité dans J. GIL, « Judios y cristianos en Hispania... », op. cit., p. 72, n. 185.

35. Présentation et bibliographie dans G. BOSSEMAN, «Pourquoi et comment lire l'Apocalypse et les Prophètes dans la péninsule Ibérique ( $\mathrm{IX}^{\mathrm{e}}-\mathrm{XI}^{\mathrm{e}}$ siècle) ? ", Questes. Revue pluridisciplinaire d'études médiévales, 38 (2018), p. 81-97.

36. MADRID, Real Academia de la Historia, $78,206 \mathrm{v}^{\circ}-210 \mathrm{v}^{\circ}$. Sur ce manuscrit, voir présentation et bibliographie dans E. RUIZ GARCíA, Catálogo de la sección de códices de la Real Academia de la Historia, Madrid, 1997, p. 395-405 ; H. de CARLos VILLAMARÍN, « El Códice de Roda (Madrid, BRAH 78) como compilación de voluntad historiográfica ", in Edad Media. Revista de historia, 12 (2011), p. 119-142.

37. A. HILHORST, "The Escorial Fragment on the Heavenly Jerusalem », in R. I. A. NIP, H. VAN DJIK et E. M. C. VAN HOUTS (dir.), Media Latinitas. A Collection of Essays to Mark the Occasion of the Retirement of L. J. Engels, Turnhout, 1996, p. 223-228.

38. J. LECLERCQ, L'amour des lettres et le désir de Dieu : initiation aux auteurs monastiques du Moyen Âge, Paris, 2008 [1 $1^{\text {re }}$ éd. 1957], p. 79-80.

39. Sur l'exégèse médiévale, l'importance de la bibliographie empêche toute exhaustivité, nous nous limiterons à mentionner entre autres travaux: C. SPICQ, Esquisse d'une histoire de l'exégèse latine au Moyen Âge, Paris, 1944 ; J. LECLERCQ, L'amour des lettres..., ibid.; H. DE LUBAC, Exégèse médiévale : les quatre sens de l'Écriture, Paris, 1993 [1 $1^{\text {re }}$ éd. 1959]; B. SMALLEY, The Study of the Bible in the Middle Ages, Oxford, 1983 ; S. CANTElLI, Angelomo e la scuola esegetica di Luxeuil, Spolète, 1990 ; G. LOBRICHON, «L'exégèse biblique, histoire d'un genre littéraire (VII ${ }^{\mathrm{e}}$-XIII ${ }^{\mathrm{e}}$ siècle) ", in ID. (dir.), La Bible au Moyen Âge, Paris, 2003, p. 55-71; C. MARTIN CHAZELLE et B. VAN NAME EDWARDS (dir.), The Study of the Bible in the Carolingian Era, Turnhout, 2003; G. DAHAN, Lire la Bible au Moyen Âge: essais d'herméneutique médiévale, Genève, 2009; I. VAN’T SPIJKER, "Introduction ", in EAD. (dir.), The Multiple Meaning of Scripture. The Role of Exegesis in Early-Christian and Medieval Culture, Leyde, 2009, p. 1-12 ; F. VAN LIERE, « Biblical Exegesis Through the Twelth Century ", in S. BOYNTON et D. J. REILLY (dir.), The Practice of the Bible in the Middle Ages: Production, Reception, and Performance in Western Christianity, New York, 2011, p.157-178; S. SHIMAHARA, Haymon d'Auxerre, exégète carolingien, Turnhout, 2013.

40. B. Smalley évoque ainsi la symbolique des nombres, l'étymologie et la digression comme les "queer laws» de l'exégèse allégorique, dont il faut s'imprégner et comprendre la logique pour aborder l'étude de la Bible au Moyen Âge, B. SMALleY, The Study of the Bible..., ibid., p. 5.

41. "The proof that the early medieval preoccupation with AM II was accompanied by an "underground" or rather, oral tradition of the sabbatical millennium appears most distinctly in the emergence of a double refrain in early medieval historiography : on the one hand, a summary of the years in each age "ab exordio mundi..." culminating in a total "ad praesentem annum"; and on the other, not uncommonly from the end of the $7^{\text {th }}$ century, a calculation of the years remaining "until the completion of this millennium" The most striking instances of these millennial countdowns appear in copies of Isidore's widely popular Six Ages, the work which ended in the last age with a total of years AM II and an explicit prohibition against eschatological calculations », R. LANDES, « Lest the Millenium... », op. cit., p. 168-169, sur Beatus, p. 193.

42. L'appréhension de la contradiction dans les sociétés pré-modernes a notamment été étudiée par C. Lévi-Strauss, qui postule que l'une des fonctions du mythe est de résoudre ou suspendre la contradiction : C. LÉVI-STRAUSS, Mythologiques, Paris, 4 vol., 1966-1971 ; ID., Anthropologie structurale, 
Paris, 1980 [1 $1^{\text {re }}$ éd. 1962], chap. 12 "Structure et dialectique», p. 254 ; voir les remarques d'A. Guerreau sur la différence entre la cohérence et la non-contradiction, et sur l'intérêt de ne pas chercher à résoudre ce qui semble «bizarre au regard du "bon sens ordinaire", i.e. contemporain ", cf. A. GUERREAU, "Le champ sémantique de l'espace dans la Vita de saint Maieul (Cluny, début du XI ${ }^{\mathrm{e}}$ siècle) », Journal des Savants, 2 (1997), p. 363-419, ici p. 414.

43. H. DE LUBAC, "À propos de la formule: "Diversi, sed non adversi" ", Recherches de science religieuse, 40 (1951), p. 27-40.

44. Dans l'interprétation de la Bible, la lecture littérale est considérée comme pouvant conduire à des contradictions, alors que les discordances des Écritures sont perçues comme la conséquence de leurs sens multiples tous en accord avec la foi : G. DAHAN, La polémique chrétienne contre le judaïsme au Moyen Âge, Paris, 1991, p.129-135; M. PARKES, «Lire, écrire, interpréter le texte. Pratiques monastiques dans le haut Moyen Âge », in G. CAVALLO, R. CHARTIER et R. BONFIL (dir.), Histoire de la lecture dans le monde occidental, Paris, 1997, p. 115-132, ici p. 126-128.

45. G. LOBRichon, La Bible au Moyen Âge..., op. cit., p. 55-71 et 76-86.

46. Ez 12, 23-28; Mt 3, 2 ; Mc 13, 30 ; Lc 21, 32 ; etc.

47. Mc $13,32-37$; Mt $24,36.42-51$ et 25,13 ; Lc. 12 ; Ap 3, 3 ; etc.

48. Mt 24,36 ; Mc $13,32$.

49. "Nos uero haec non secundum litteram, sed secundum sensum intelligere debemus [...] unde manifestum est quod filius sciuit, sed discipulis indicare noluit, ut semper de morte suspecti et incerti spem in seculo non haberent ", BEATUS DE LIÉBANA, Tractatus de Apocalipsin, éd. R. GRYSON, t. 2, IV, 5, § 22-25, p. 520-521.

50. ALVARE, Epistula VIII, in Scriptores Muzarabici saeculi VIII-XI, éd. J. GIL FERNÁNDEZ, Turnhout, 2020, t. 1, p. 499-508 (Corpus Christianorum, Continuatio Mediaevalis, 65A).

51. J. LECLERCQ, L'amour des lettres..., op. cit.; sur la lectio diuina, voir aussi : «Lectio diuina », in Dictionnaire de spiritualité, t. 9, col. 470-510 ; B. CALATI, « La Lectio divina nella tradizione monastica benedettina ", Benedictina, 28 (1981), p. 407-438; W. WITTERS, " Travail et lectio divina dans le monachisme de St. Benoit ", in Atti del $7^{\circ}$ Congresso internazionale di studi sull'Alto Medioevo, Norcia, Subiaco, Cassino, Montecassino, 29 settembre-5 ottobre 1980, Spolète, 1982, t.2/2, p. 551-561; D. ROBERTSON, «Lectio divina » : The Medieval Experience of Reading, Trappist, Ky., 2011.

52. M. CARRUTHERS, The Book of Memory: a Study of Memory in Medieval Culture, Cambridge, 1992 ; EAD., The Craft of Thought: Meditation, Rhetoric, and the Making of Images, 400-1200, Cambridge, 1998, trad. fr. Machina memorialis : méditation, rhétorique et fabrication des images au Moyen Âge, trad. fr. F. DURAND-BOGAERT, Paris, 2002.

53. В. Ітоск, Lire, une ascèse ? Lecture ascétique et lecture esthétique dans la culture occidentale, trad. fr. C. CARRAUD, Grenoble, 2008, p. 81.

54. Ad concupiscendum regnum perdisti sensum, aperi sensum et adquires regnum, repr., citation et trad. ang. dans C. BROWN, «Remember the Hand: Bodies and Bookmaking in Early Medieval Spain ", in Word \& Image, 27/3 (2011), p. 262-278, ici p. 272-273, qui note le parallèle avec Sap. 6, 21.

55. Mary Carruthers a montré que la machina memorialis s'appuie sur l'allégorie qui va « élever les pensées du lecteur » par une succession d'associations passant du chiffre à la lettre et à l'image. Ainsi, la capacité d'association permet de «découvrir et de rassembler le matériau qui se dissimule dans les recoins de la mémoire", et de mettre à jour un sens "secret", cf. M. CARRUTHERS, Machina Memorialis..., op. cit., p. 212-213.

56. En plus des travaux déjà cités, voir: F. BOUCHET, «Les cinq sens du lecteur médiéval », in K. UELTSCHI-COURChINOUX (dir.), L'univers du livre médiéval. Substance, lettre, signe, Genève, 2014, p. 289-306.

57. In omnibus operibus tuis memorare nouissima tua, et in aeternum non peccabis, cité par BEATUS, Tractatus, IV, 5, § 32, t. 2, p. 523. 
58. M. CARRUTHERS, The Craft of Thought..., op. cit., p.66-68. Voir aussi sur «l'exercice de Jérusalem », J. LECLERCQ, L'amour des lettres..., op. cit., p. 56-58.

59. M. CARRUTHERS, The Craft of Thought..., ibid., p. 94.

60. Sur les livres supports de la lectio diuina et plus généralement sur les indications la concernant dans les règles monastiques, voir A. M. MUNDó, «Las reglas monásticas latinas del siglo VI y la lectio diuina », Studia monastica, 9 (1967), p. 229-255.

61. "Quae quamuis omnibus nota sint, qui per amplitudinem scripturarum percurrunt, facilius tamen ad memoriam redeunt, dum breui sermone leguntur », BEATUS, « Prologus », Tractatus, t. 1, p. 2.

62. J. FONTAINE, « Fuentes y tradiciones... », op. cit., p. 88-98 ; M. MENTRÉ, La peinture mozarabe : un art chrétien hispanique autour de l'an 1000, Paris, 1995 ; M. CARRUTHERS, The Craft of Thought..., op. cit., p. 152-155 ; P. GAUTIER DALCHÉ, « De la glose à la contemplation : place et fonction de la carte dans les manuscrits du haut Moyen Âge ", in Settimane di studio del Centro Italiano di Studi sull'Alto Medioevo, Spolète, 1994, p. 693-764 ; voir aussi sur ce mécanisme, B. STоск, Lire, une ascèse? Lecture ascétique..., op. cit., p. 89-90.

63. В. Атоск, Lire, une ascèse ? Lecture ascétique..., ibid., p. 81-82.

64. « Factum est silentium in celo quasi semihora, id est, serui Dei, qui ab omni seculari actione uacant et in contemplatione hic incipiunt uitam eternam degustare ", BEATUS, Tractatus, prolegomena, 4 , § 22, t. 1, p. 14-15.

65. "Homines sunt ratione capaces, preterita recordant, presentia ordinant, et futura preuident, et quicquid de die in diem faciunt, incessanter pensant, ne ad deteriora umquam uel in opere uel in cogitatione decidant; non dant somnum oculis, sed die et nocte in lege domini meditant ", BEATUS, Tractatus, pr. II, 6, § 4, t. 1, p. 178. Il reprend le thème dans un autre passage du Commentaire: « Et quid facit iste homo, nisi omnes sensos in capite portet, ita ut oculis uideat, id est preterita recordet, presentia ordinet, et semper futura preuideat, et oculos testamentorum dei, legis et euangelii, medullitus inuestiget... [s'ensuit un développement sur les sens] », II, 5, § 22, p. 310.

\section{RÉSUMÉS}

Prenant position dans le débat historiographique sur la réalité des peurs millénaristes à l'approche de l'an 800 et de l'an 1000, cet article propose une relecture de quelques textes abordant la fin des temps composés dans la péninsule Ibérique au haut Moyen Âge. En observant leur composition et leur contexte de transmission, il s'agit de montrer que ces textes ont assumé des fonctions érudites et savantes - compilation de savoirs, inculcation de la doctrine eschatologique, enseignement, etc. -, a priori détachées de toute attente angoissée de la fin des temps. Objet de savoir autant que de croyance, l'eschatologie est principalement abordée par les lettrés à travers l'exégèse des prophéties; l'étude s'attache en outre à restituer les dimensions spirituelle et méditative dans lesquelles les textes sur la fin des temps étaient lus et étudiés.

Taking a stance in the historiographical debate on the reality of millenarian fears at the approach of the year 800 and the year 1000, this article offers a reinterpretation of some texts on the end of times in the Iberian Peninsula in the Early Middle Ages. By observing their composition and their context of transmission, the objective is to show that these texts have assumed erudite and scholarly functions - compilation of knowledge, inculcation of eschatological doctrine, teaching, etc. - a priori detached from any fearful expectation of the last 
days. Object of knowledge as much as of belief, eschatology is mainly approached by medieval scholars through the exegesis of prophecies; the study furthermore endeavors to restore the spiritual and meditative dimension in which the texts on the end of times were read and studied.

INDEX

Mots-clés : savoir, enseignement, numérologie, exégèse, méditation, manuscrits, Beatus de Liébana, Indiculus de aduentu Henoch et Eliae, Interrogatio de nobissimo

\section{AUTEUR}

\section{GAELLE BOSSEMAN}

ATER, université Toulouse-Jean Jaurès 